\title{
Adaptive Synchronization Control of Multiple Vessels with Switching Communication Topologies and Time Delay
}

\author{
Fuguang Ding, ${ }^{1}$ Yanqin $\mathrm{Ma},{ }^{1}$ Yuanwei Zhou, ${ }^{2}$ and Jiangjun $\mathrm{Li}^{1}$ \\ ${ }^{1}$ College of Automation, Harbin Engineering University, Harbin 150001, China \\ ${ }^{2}$ Shandong Hyundai Wia Automotive Engine Co., Ltd., Rizhao 276800, China \\ Correspondence should be addressed to Yanqin Ma; mayanqin@hrbeu.edu.cn
}

Received 4 July 2014; Revised 12 November 2014; Accepted 13 November 2014

Academic Editor: Mehmet Onder Efe

Copyright (c) 2015 Fuguang Ding et al. This is an open access article distributed under the Creative Commons Attribution License, which permits unrestricted use, distribution, and reproduction in any medium, provided the original work is properly cited.

\begin{abstract}
Recently, synchronization movement control of multiple vessels has been studied broadly. In most of the studies, the communication network among vessels is considered to be fixed and the time delay is often ignored. However, the communication network among vessels maybe vary because of switching of different tasks, and the time delay is necessary to be considered when the communication network is unreliable. In this paper, the synchronization movement of multiple vessels with switching connected communication topologies is studied, and an adaptive synchronization control algorithm that is based on backstepping sliding mode control is proposed. The control algorithm is achieved by defining cross coupling error which is combination of the trajectory tracking error and velocity tracking error. And an adaptive control term is used to estimate the external disturbances, so that the unknown external disturbances can be compensated. Furthermore, the robustness of the control law to time-varying time delay is also discussed. At last, some simulations are carried out to validate the effectiveness of the proposed synchronization control algorithm.
\end{abstract}

\section{Introduction}

Recently, the applications of synchronization movement control of multiple vessels are increasing. For example, in underway replenishment, its need to control supply vessels maintains the same state with the receiving vessel to insure safety. And another example is a group of vessels performing the seabed mapping operations together, which can implement larger area in shorter time compared with a single vessel. Multiple vessels work together not only improving operation performance but also reducing difficulty. And this paper mainly discusses the synchronization movement control of multiple vessels.

In recent years, synchronization control of multiple agents has been extensively studied in different fields, such as robot systems $[1,2]$, chaotic systems consensus $[3,4]$, and coordinated formation control of aircraft and spacecraft [5, 6]. As the development of synchronization control of multiple agents, there are several synchronization control approaches which are proposed. For example, distributed cooperative attitude synchronization control approach has been discussed in [7]. And adaptive control is a classical strategy used to address synchronization movement $[8,9]$. Besides, using cascaded system theory and graph theory, a distributed attitude cooperative control scheme is studied in [10]. Compared with the earlier work in computational load and required states, Chung and Slotine proposes a simple synchronization framework to achieve cooperative movement of multiple agents [11]. In the presence of uncertainties of underactuated autonomous surface vehicles and ocean disturbances, a robust adaptive dynamic surface control law is proposed in [12]. There are some other synchronization movement control methods, such as backstepping control design [13, 14], artificial potential approach [15], leader-follower network strategy [16, 17], and methods that are based on graph theory $[18,19]$. The main traits of the aforementioned articles are that they assume the communication topology is fixed without considering time delay.

Switching communication topologies and time delay are two fundamental realities in the communication among vessels, but these factors are usually ignored in [20-22]. In recent years, switching communication topologies or time delay has 
been investigated [23-25], and time delay in the communication network is usually assumed to be a constant. However, in multiple-vessel network, because the bandwidth of communication is limited, communication topology among vessels may be varied when the marine task changes. And the time delay cannot be a constant because of the varying relative position among vessels and unknown external statistics. Thus, it is necessary to design a synchronization controller for multiple vessels with switching communication topologies and time delay. Moreover, for the surface vessels, which often encounter external disturbances, and the disturbances are usually difficult to model because of the disturbances varying with the complex ocean circumstance. So the synchronization movement control law should be robust to unknown disturbances and an adaptive control is useful to solve this problem [26-28].

In view of the above reasons, the main innovation of this paper can be drawn as follows: an adaptive synchronization movement control law that is based on backstepping sliding mode control for multiple vessels with switching communication topologies is proposed. Different from the traditional controller design approach that maybe suffers difficulties to determine the Lyapunov function, the backstepping-based design method provides us with an appropriate Lyapunov function simply and ensures the stability of closed-loop system, and the sliding mode method shows robustness to external disturbances and system uncertainty; the combination of backstepping and sliding mode takes both advantages of the two methods, and an adaptive term is introduced to improve the synchronization control algorithm. Moreover, the directed strongly connected communication topology may be not balanced, which means one vessel can receive information from the neighbors and not necessarily share its own information with the neighbors, and compared to the existing studies, the requirement on the communication is relaxed. And cross coupling error using trajectory tracking error and velocity tracking error is defined, and the cross coupling error is introduced into the switched function. Furthermore, in the presence of time delay, the designed synchronization control strategy is improved, and the synchronization control method is robust to time delay.

The arrangement of this paper is as follows. In Section 2, the basic knowledge for graph theory and the mathematical model of vessels are given. The adaptive synchronization control approach with switching communication topologies and time delay is discussed in Section 3. In Section 4, some simulations are carried out to validate the proposed control algorithm. At last, conclusions and constructive prospects are drawn in Section 5.

\section{Preliminaries}

2.1. Graph Theory. For the multiple vessels, the communication topology and information exchange among vessels can be described by a graph. Let $G=\{V, E\}$ describe the information exchanges among vessels, which consists of nodes $V(G)=\left\{v_{1}, v_{2}, \ldots, v_{n}\right\}$ and edges $E(G) \subseteq V \times V$, and $E(G)$ composes edges where joint nodes come from $V$.
A node in the graph represents a vessel. The edges represent the information exchange links among the vessels. Node $j$ is a neighbor of node $i$, if $\left(v_{i}, v_{j}\right) \in E(G)$. Let the set of the neighbors of node $i$ denoted by $N_{i}=\left\{v_{j} \in V /\left(v_{i}, v_{j}\right) \in E\right\}$. Assume matrix $A$ is the adjacency matrix of graph $G$, and $a_{i j} \in A$ is defined as $a_{i j}>0$, if $\left(v_{i}, v_{j}\right) \in E$, otherwise, $a_{i j}=0$, if $\left(v_{i}, v_{j}\right) \notin E$. Define $d_{i}=\sum_{j \neq i} a_{i j}, D=\operatorname{diag}\left\{d_{i}\right\}$; then the Laplacian matrix of the weighted graph $G$ can be noted $L=D-A$. For all $\left(v_{i}, v_{j}\right) \in E(G)$, if $\left(v_{j}, v_{i}\right) \in E(G)$, then the graph is called undirected. Otherwise, it is directed.

Define a set of $m$ communication topology graphs $G=$ $\left\{\begin{array}{lll}G_{1} & \cdots & G_{m}\end{array}\right\}$. And the nodes of graph $G_{t}$ are the same, but the edge sets are different. Therefore, the Laplacian matrix of graph $G_{t}$ is denoted by $L_{t}$. It is necessary to satisfy that all the communication topologies are connected and $L_{t}$ is a positive semidefinite matrix.

If the communication topology is connected, then define $\forall x=\left[\begin{array}{lll}x_{1}^{T} & \cdots & x_{m}^{T}\end{array}\right]^{T}$ is a column stack vector, and $x$ satisfies $x^{T}(L \otimes I) x=(1 / 2) \sum_{i=1}^{n} \sum_{j=1}^{n} a_{i j}\left(x_{i}-x_{j}\right)$.

2.2. Mathematical Model of Vessel. The 3-DOF surface motion model of the vessel can be described as

$$
\dot{\eta}=R(\psi) \nu, \quad M \dot{v}+C(\nu) \nu+D(\nu) \nu=\tau_{v}+\tau_{v d},
$$

where $\eta=[n, e, \psi]^{T}$ is the position and heading in the earthfixed reference frame and $v=[u, v, r]^{T}$ is the velocity with regard to the body-fixed reference frame. $R(\psi)$ is a rotation matrix, which can be written as

$$
R(\psi)=\left[\begin{array}{ccc}
\cos (\psi) & -\sin (\psi) & 0 \\
\sin (\psi) & \cos (\psi) & 0 \\
0 & 0 & 1
\end{array}\right]
$$

where $M$ is a positive definite inertia mass matrix and $M=$ $M^{T}$. $C(v)$ represents the hydrodynamic Coriolis and centripetal matrix. $D(v)$ denotes damping matrix. $\tau_{v}$ illustrates the control forces and torques input. $\tau_{v d}$ are the external disturbances.

The motion mathematical model of the vessel in the earth-fixed reference frame is

$$
\begin{gathered}
\nu=R^{T}(\psi) \dot{\eta}, \\
M(\eta) \ddot{\eta}+C(\eta, \dot{\eta}) \dot{\eta}+D(\eta, \dot{\eta}) \dot{\eta}=\tau(\eta)+\tau_{d},
\end{gathered}
$$

where the relation of the conversion yields

$$
\begin{gathered}
M(\eta)=R^{-T}(\psi) M R^{-1}(\psi), \\
C(\eta, \dot{\eta})=R^{-T}(\psi) C(v) R^{-1}(\psi) \\
-R^{-T}(\psi) M R^{-1}(\psi) \dot{R}^{-1}(\psi), \\
D(\eta, \dot{\eta})=R^{-T}(\psi) D(v) R^{-1}(\psi), \\
\tau(\eta)=R^{-T}(\psi) \tau_{v}, \quad \tau_{d}=R^{-T}(\psi) \tau_{v d} .
\end{gathered}
$$

The vessel model (3) holds the following properties. 
Property 1. The inertia matrix $M(\eta)$ is symmetric, positive definite, and bounded as

$$
\lambda_{\min }(M) \leq\|M(\eta)\| \leq \lambda_{\max }(M) .
$$

Property 2. The damping matrix $D(\eta, \dot{\eta})$ satisfies

$$
\eta^{T} D(\eta, \dot{\eta}) \eta=\frac{\eta^{T}\left(D(\eta, \dot{\eta})+D(\eta, \dot{\eta})^{T}\right) \eta}{2} \geq 0 .
$$

\section{Adaptive Synchronization Control Design}

3.1. Adaptive Synchronization Control with Switching Communication Topologies. The objective of this section is to develop a control method for achieving state synchronization of multiple vessels while tracking time-varying trajectories $\eta_{i d}$, and $i=\{1,2, \ldots, n\}$, in the presence of switching communication topologies, and a new backstepping sliding mode synchronization controller is designed.

The model of vessel $i$ in a compact form yields

$$
v_{i}=R_{i}^{T}(\psi) \dot{\eta}_{i}, \quad \ddot{\eta}_{i}=M_{i}^{-1}\left(\tau_{i}+\tau_{i d}-C_{i}-D_{i}\right) .
$$

Define the trajectory tracking error of vessel $i$ as

$$
\widetilde{\eta}_{i}=\eta_{i}-\eta_{i d}
$$

Take the derivative of $\tilde{\eta}_{i}$ as

$$
\dot{\vec{\eta}}_{i}=\dot{\eta}_{i}-\dot{\eta}_{i d}
$$

Define the stabilizing function for vessel $i$ as

$$
\alpha_{i}=\lambda \tilde{\eta}_{i}
$$

where $\lambda \in R^{3 \times 3}$ is a diagonal positive definite matrix.

Define the velocity tracking error of vessel $i$ as

$$
\widetilde{v}_{i}=\dot{\tilde{\eta}}_{i}+\lambda \tilde{\eta}_{i}
$$

The switched function of vessel $i$ is chosen as

$$
\sigma_{i}=\tilde{\eta}_{i}+\widetilde{v}_{i}=\dot{\vec{\eta}}_{i}+\Lambda \tilde{\eta}_{i}
$$

where $\Lambda=\lambda+I_{3 \times 3}$ is a diagonal positive definite matrix.

Define a new parameter $\sigma_{i j}$ as

$$
\sigma_{i j}=\sigma_{i}-\sigma_{j}
$$

The vessels are said to synchronize if

$$
\lim _{t \rightarrow \infty}\left\|\sigma_{i j}\right\|=0, \quad \forall i, j \in N_{i} .
$$

With vessel model (7), we can get

$$
\begin{aligned}
M_{i} \dot{\sigma}_{i} & =M_{i}\left(\ddot{\tilde{\eta}}_{i}+\Lambda \dot{\tilde{\eta}}_{i}\right)=M_{i}\left(\ddot{\eta}_{i}-\ddot{\eta}_{i d}\right)+M_{i} \Lambda \dot{\tilde{\eta}}_{i} \\
& =\tau_{i}+\tau_{i d}-C_{i} \dot{\eta}_{i}-D_{i} \dot{\eta}_{i}-M_{i} \ddot{\eta}_{i d}+M_{i} \Lambda \dot{\vec{\eta}}_{i} .
\end{aligned}
$$

In order to prove stability of $m$ switching communication topologies, define a common Lyapunov function for all $m$ communication topologies.
Define the first Lyapunov function as

$$
V_{1}=\frac{1}{2} \sum_{i=1}^{n} \widetilde{\eta}_{i}^{T} \widetilde{\eta}_{i}
$$

The time derivative of (16) yields

$$
\dot{V}_{1}=\sum_{i=1}^{n} \widetilde{\eta}_{i}^{T} \dot{\vec{\eta}}_{i}=\sum_{i=1}^{n}\left(\tilde{\eta}_{i}^{T} \sigma_{i}-\tilde{\eta}_{i}^{T} \Lambda \widetilde{\eta}_{i}\right)
$$

Choose the second Lyapunov function as

$$
V_{2}=V_{1}+\frac{1}{2} \sum_{i=1}^{n} \sigma_{i}^{T} \sigma_{i}
$$

Therefore, the time derivative of (18) yields

$$
\begin{aligned}
\dot{V}_{2}= & \dot{V}_{1}+\sum_{i=1}^{n} \sigma_{i}^{T} \dot{\sigma}_{i} \\
= & \sum_{i=1}^{n}\left(\widetilde{\eta}_{i}^{T} \sigma_{i}-\widetilde{\eta}_{i}^{T} \Lambda \widetilde{\eta}_{i}\right) \\
& +\sum_{i=1}^{n} \sigma_{i}^{T} M_{i}^{-1}\left(\tau_{i}+\tau_{i d}-C_{i} \dot{\eta}_{i}-D_{i} \dot{\eta}_{i}-M_{i} \ddot{\eta}_{i d}+M_{i} \Lambda \dot{\vec{\eta}}_{i}\right) .
\end{aligned}
$$

Let the coupling control law for vessel $i$ be

$$
f_{i}=a_{i j} \sum_{j=1}^{n}\left(\sigma_{i}-\sigma_{j}\right)=a_{i j} \sum_{j=1}^{n} \sigma_{i j} .
$$

Choose the synchronization control input as

$$
\begin{aligned}
\tau_{i}= & C_{i} \dot{\eta}_{i}+D_{i} \dot{\eta}_{i}-\widehat{\tau}_{i d}+M_{i} \ddot{\eta}_{i d}-M_{i} \Lambda \dot{\tilde{\eta}}_{i} \\
& -M_{i}\left(\widetilde{\eta}_{i}+\sigma_{i}+f_{i}+\operatorname{sign}\left(\sigma_{i}\right)\right),
\end{aligned}
$$

where $\widehat{\tau}_{i d}$ is the estimate of external disturbances and $a_{i j}$ is the element of weighted adjacency matrix $A$.

Define the third Lyapunov function as

$$
V_{3}=V_{2}+\sum_{i=1}^{n} \frac{1}{2 \kappa_{i}} \tilde{\tau}_{i d}^{T} \tilde{\tau}_{i d}
$$

where $\widetilde{\tau}_{i d}=\tau_{i d}-\widehat{\tau}_{i d}$ and $\kappa_{i}$ is a positive number.

Assume the disturbances $\tau_{i d}$ are unknown in advance and $\tau_{i d}$ vary slowly, which means $\dot{\tau}_{i d}=0$.

The time derivative of (22) yields

$$
\begin{aligned}
\dot{V}_{3}= & \dot{V}_{2}-\sum_{i=1}^{n} \frac{1}{\kappa_{i}} \tilde{\tau}_{i d}^{T} \dot{\hat{\tau}}_{i d} \\
= & \sum_{i=1}^{n}\left(\widetilde{\eta}_{i}^{T} \sigma_{i}-\tilde{\eta}_{i}^{T} \Lambda \tilde{\eta}_{i}-\sigma_{i}^{T}\left(\widetilde{\eta}_{i}+\sigma_{i}+\operatorname{sign}\left(\sigma_{i}\right)\right)\right) \\
& +\sum_{i=1}^{n}\left(\sigma_{i}^{T} M_{i}^{-1} \widetilde{\tau}_{i d}-\sigma_{i}^{T} a_{i j} \sum_{j=1}^{n} \sigma_{i j}-\frac{1}{\kappa_{i}} \widetilde{\tau}_{i d}^{T} \dot{\widehat{\tau}}_{i d}\right)
\end{aligned}
$$




$$
\begin{aligned}
& =\sum_{i=1}^{n}\left(-\tilde{\eta}_{i}^{T} \Lambda \tilde{\eta}_{i}-\sigma_{i}^{T} \sigma_{i}-\sigma_{i}^{T} \operatorname{sign}\left(\sigma_{i}\right)\right) \\
& +\sum_{i=1}^{n}\left(\tilde{v}_{i}^{T} M_{i}^{-1} \tilde{\tau}_{i d}-\sigma_{i}^{T} a_{i j} \sum_{j=1}^{n} \sigma_{i j}-\frac{1}{\kappa_{i}} \tilde{\tau}_{i d}^{T} \dot{\vec{\tau}}_{i d}\right) \\
& =-\sum_{i=1}^{n}\left(\tilde{\eta}_{i}^{T} \Lambda \tilde{\eta}_{i}+\sigma_{i}^{T} \sigma_{i}+\sigma_{i}^{T} \operatorname{sign}\left(\sigma_{i}\right)\right. \\
& \left.+\frac{\tilde{\tau}_{i d}^{T}}{\kappa_{i}}\left(-\kappa_{i} M_{i}^{-T} \sigma_{i}+\dot{\hat{\tau}}_{i d}\right)\right)-\frac{1}{2} \sum_{i=1}^{n} \sum_{j=1}^{n} \sigma_{i j}^{T} a_{i j} \sigma_{i j},
\end{aligned}
$$

where

$$
\begin{aligned}
\frac{1}{2} \sum_{i=1}^{n} \sum_{j=1}^{n} \sigma_{i j}^{T} a_{i j} \sigma_{i j} & =\frac{1}{2} \sum_{i=1}^{n} \sum_{j=1}^{n}\left(\sigma_{i}^{T} a_{i j} \sigma_{i j}-\sigma_{j}^{T} a_{i j} \sigma_{i j}\right) \\
& =\frac{1}{2} \sum_{i=1}^{n} \sum_{j=1}^{n} \sigma_{i}^{T} a_{i j} \sigma_{i j}+\frac{1}{2} \sum_{i=1}^{n} \sum_{j=1}^{n} \sigma_{j}^{T} a_{i j} \sigma_{j i} \\
& =\sum_{i=1}^{n} \sum_{j=1}^{n} \sigma_{i}^{T} a_{i j} \sigma_{i j} .
\end{aligned}
$$

Then, we adopt an adaptive term to estimate the disturbances. Define the adaptive control law of $\widehat{\tau}_{i d}$ as

$$
\dot{\hat{\tau}}_{i d}=\kappa_{i} M_{i}^{-T} \sigma_{i}
$$

where $\kappa_{i}$ is a positive constant.

With (25) and (23), we can obtain

$$
\begin{aligned}
\dot{V}_{3}= & -\sum_{i=1}^{n}\left(\tilde{\eta}_{i}^{T} \Lambda \tilde{\eta}_{i}+\sigma_{i}^{T} \sigma_{i}+\sigma_{i}^{T} \operatorname{sign}\left(\sigma_{i}\right)\right) \\
& -\frac{1}{2} \sum_{i=1}^{n} \sum_{j=1}^{n} \sigma_{i j}^{T} a_{i j} \sigma_{i j} \\
= & -\sum_{i=1}^{n}\left(\tilde{\eta}_{i}^{T} \Lambda \tilde{\eta}_{i}+\sigma_{i}^{T} \sigma_{i}+\sigma_{i}^{T} \operatorname{sign}\left(\sigma_{i}\right)\right) \\
& -\sigma^{T}\left(L_{t} \otimes I_{k}\right) \sigma \\
\leq & -\sum_{i=1}^{n}\left(\tilde{\eta}_{i}^{T} \Lambda \tilde{\eta}_{i}+\sigma_{i}^{T} \sigma_{i}+\left|\sigma_{i}\right|\right)-\sigma^{T}\left(L_{t} \otimes I_{k}\right) \sigma,
\end{aligned}
$$

where $t \in\{1, \ldots, m\}$ represents the $t$ th communication topology and $\sigma=\left[\begin{array}{lll}\sigma_{1}^{T} & \cdots & \sigma_{n}^{T}\end{array}\right]^{T}$.

Consider the communication topologies are connected; then (26) is a negative definite function, and in view of the Lyapunov function (22) is a strict common function for the switched system. Therefore, we can get $\widetilde{\eta}_{i} \rightarrow 0, \sigma_{i} \rightarrow 0$, and $\sigma_{i j} \rightarrow 0$ as $t \rightarrow \infty$ with the arbitrary switching among the $m$ communication topologies; with (8) and (12), we can get $\eta_{i} \rightarrow \eta_{i d}, \dot{\eta}_{i} \rightarrow \dot{\eta}_{i d}$, and $\widetilde{v} \rightarrow 0$ as $t \rightarrow \infty$; the velocity tracking error and trajectory tracking error approached zero asymptotically.
With (12) and (13), $\sigma_{i j}$ yields

$$
\begin{aligned}
\sigma_{i j} & =\sigma_{i}-\sigma_{j}=\dot{\tilde{\eta}}_{i}+\Lambda \tilde{\eta}_{i}-\dot{\tilde{\eta}}_{j}-\Lambda \tilde{\eta}_{j} \\
& =\dot{\eta}_{i}-\dot{\eta}_{j}+\dot{l}_{i j}+\Lambda\left(\eta_{i}-\eta_{j}+l_{i j}\right) \\
& =\dot{\eta}_{i j}+\dot{l}_{i j}+\Lambda\left(\eta_{i j}+l_{i j}\right)=\dot{\mu}_{i j}+\Lambda \mu_{i j},
\end{aligned}
$$

where $\eta_{i j}=\eta_{i}-\eta_{j}$ denotes the synchronization position error between vessel $i$ and vessel $j$ and $l_{i j}=\eta_{i d}-\eta_{j d}$ is the desired relative position between vessel $i$ and vessel $j$. And note $\mu_{i j}=$ $\eta_{i j}+l_{i j}$. From (27), we can know that (27) represents a linear exponentially stable system with the input $\sigma_{i j}$, as $\sigma_{i j} \rightarrow 0$ and $\mu_{i j}$ is bounded. Then, it can be obtained that $\lim _{t \rightarrow \infty}\left\|\mu_{i j}\right\|=0$, which means $\eta_{i}-\eta_{j} \rightarrow l_{i j}, \dot{\eta}_{i}-\dot{\eta}_{j} \rightarrow \dot{l}_{i j}$. Therefore, the synchronization position error and synchronization velocity error approach zero asymptotically; that is, the vessels achieve state synchronization.

Theorem 1. Consider the model of vessel $i$ described by (7) with the synchronization control law (21) and adaptive control law (25). If the communication topologies are connected, then the synchronization errors and the tracking errors are uniformly ultimately bounded in spite of switching communication topologies and unknown disturbances.

3.2. Adaptive Synchronization Control with Time Delay. In view of unreliable communication networks among multiple vessels, time-varying time delay is introduced to describe the condition. In this section, $T_{i j}$ denotes the time delay from vessel $i$ to vessel $j$, and it is assumed that time delay is bounded and continuously differentiable, which means $0<$ $T_{i j} \leq T_{m}<\infty, \dot{T}_{i j} \leq T^{m}<1, \forall i=1, \ldots, n$.

In the presence of time delay, the vessels are considered to be delayed synchronization if

$$
\lim _{t \rightarrow \infty}\left(\sigma_{j}\left(t-T_{j i}\right)-\sigma_{i}(t)\right)=0, \quad \forall i, j \in N_{i} .
$$

To achieve delayed synchronization, define a positive constant gain as

$$
d_{j i}^{2} \leq 1-T^{m}
$$

Similar to the front section, the procedure yields the following.

Define the first Lyapunov function as

$$
V_{1}=\frac{1}{2} \sum_{i=1}^{n} \tilde{\eta}_{i}^{T} \tilde{\eta}_{i}
$$

The time derivative of (30) yields

$$
\dot{V}_{1}=\sum_{i=1}^{n} \widetilde{\eta}_{i}^{T} \dot{\vec{\eta}}_{i}=\sum_{i=1}^{n}\left(\tilde{\eta}_{i}^{T} \sigma_{i}-\tilde{\eta}_{i}^{T} \Lambda \tilde{\eta}_{i}\right)
$$

Choose the second Lyapunov function as

$$
V_{2}=V_{1}+\frac{1}{2} \sum_{i=1}^{n} \sigma_{i}^{T} \sigma_{i}+\frac{1}{2} \sum_{i=1}^{n} \sum_{j=1}^{n} a_{i j} \int_{t-T_{j i}}^{t} \sigma_{j}^{T}(\varepsilon) \sigma_{j}(\varepsilon) d \varepsilon .
$$


Therefore, the time derivative of (32) yields

$$
\begin{aligned}
\dot{V}_{2} \leq & \dot{V}_{1}+\sum_{i=1}^{n} \sigma_{i}^{T} \dot{\sigma}_{i} \\
& +\frac{1}{2} \sum_{i=1}^{n} \sum_{j=1}^{n} a_{i j}\left(\sigma_{j}^{T} \sigma_{j}-\left(1-\dot{T}_{j i}\right) \sigma_{j}^{T}\left(t-T_{j i}\right) \sigma_{j}\left(t-T_{j i}\right)\right) \\
\leq & \sum_{i=1}^{n}\left(\tilde{\eta}_{i}^{T} \sigma_{i}-\widetilde{\eta}_{i}^{T} \Lambda \widetilde{\eta}_{i}\right) \\
& +\sum_{i=1}^{n} \sigma_{i}^{T} M_{i}^{-1}\left(\tau_{i}+\tau_{i d}-C_{i} \dot{\eta}_{i}-D_{i} \dot{\eta}_{i}-M_{i} \ddot{\eta}_{i d}+M_{i} \Lambda \dot{\tilde{\eta}}_{i}\right) \\
& +\frac{1}{2} \sum_{i=1}^{n} \sum_{j=1}^{n} a_{i j}\left(\sigma_{j}^{T} \sigma_{j}-\left(1-\dot{T}_{j i}\right) \sigma_{j}^{T}\left(t-T_{j i}\right) \sigma_{j}\left(t-T_{j i}\right)\right) .
\end{aligned}
$$

Choose the delayed synchronization control input as

$$
\begin{aligned}
\tau_{i}= & C_{i} \dot{\eta}_{i}+D_{i} \dot{\eta}_{i}-\widehat{\tau}_{i d}+M_{i} \ddot{\eta}_{i d}-M_{i} \Lambda \dot{\vec{\eta}}_{i} \\
& -M_{i}\left(\widetilde{\eta}_{i}+\sigma_{i}+\operatorname{sign}\left(\sigma_{i}\right)\right) \\
& -M_{i} a_{i j} \sum_{j=1}^{n}\left(d_{j i}^{2} \sigma_{j}\left(t-T_{j i}\right)-\frac{d_{j i}^{2}+1}{2} \sigma_{i}\right),
\end{aligned}
$$

where $\widehat{\tau}_{i d}$ is the estimate of the disturbances.

Define the third Lyapunov function as

$$
V_{3}=V_{2}+\sum_{i=1}^{n} \frac{1}{2 \kappa_{i}} \tilde{\tau}_{i d}^{T} \tilde{\tau}_{i d}
$$

where $\widetilde{\tau}_{i d}=\tau_{i d}-\widehat{\tau}_{i d}$, and $\kappa_{i}$ is a positive number.

The time derivative of (35) yields

$$
\begin{aligned}
\dot{V}_{3}= & \dot{V}_{2}-\sum_{i=1}^{n} \frac{1}{\kappa_{i}} \widetilde{\tau}_{i d}^{T} \dot{\vec{\tau}}_{i d} \\
\leq & \sum_{i=1}^{n}\left(-\widetilde{\eta}_{i}^{T} \Lambda \widetilde{\eta}_{i}+\sigma_{i}^{T} M_{i}^{-1} \tilde{\tau}_{i d}-\sigma_{i}^{T} \operatorname{sign}\left(\sigma_{i}\right)\right) \\
& +\sum_{i=1}^{n} \sigma_{i}^{T} a_{i j} \sum_{j=1}^{n}\left(d_{j i}^{2} \sigma_{j}\left(t-T_{j i}\right)-\frac{d_{j i}^{2}+1}{2} \sigma_{i}\right) \\
& -\sum_{i=1}^{n} \frac{1}{\kappa_{i}} \widetilde{\tau}_{i d}^{T} \dot{\hat{\tau}}_{i d} s \\
& +\frac{1}{2} \sum_{i=1}^{n} \sum_{j=1}^{n} a_{i j}\left(\sigma_{j}^{T} \sigma_{j}-\left(1-\dot{T}_{j i}\right) \sigma_{j}^{T}\left(t-T_{j i}\right) \sigma_{j}\left(t-T_{j i}\right)\right) \\
\leq & \sum_{i=1}^{n}\left(-\tilde{\eta}_{i}^{T} \Lambda \tilde{\eta}_{i}+\sigma_{i}^{T} M_{i}^{-1} \widetilde{\tau}_{i d}-\sigma_{i}^{T} \operatorname{sign}\left(\sigma_{i}\right)-\frac{1}{\kappa_{i}} \tilde{\tau}_{i d}^{T} \dot{\vec{\tau}}_{i d}\right) \\
& +\frac{a_{i j}}{2} \sum_{i=1}^{n} \sum_{j=1}^{n}\left(2 \sigma_{i}^{T} d_{j i}^{2} \sigma_{j}\left(t-T_{j i}\right)-\sigma_{i}^{T} d_{j i}^{2} \sigma_{i}-\sigma_{i}^{T} \sigma_{i}\right) \\
& +\frac{a_{i j}}{2} \sum_{i=1}^{n} \sum_{j=1}^{n}\left(\sigma_{j}^{T} \sigma_{j}-\left(1-\dot{T}_{j i}\right) \sigma_{j}^{T}\left(t-T_{j i}\right) \sigma_{j}\left(t-T_{j i}\right)\right)
\end{aligned}
$$

$$
\begin{aligned}
\leq & \sum_{i=1}^{n}\left(-\widetilde{\eta}_{i}^{T} \Lambda \widetilde{\eta}_{i}+\sigma_{i}^{T} M_{i}^{-1} \tilde{\tau}_{i d}-\sigma_{i}^{T} \operatorname{sign}\left(\sigma_{i}\right)-\frac{1}{\kappa_{i}} \widetilde{\tau}_{i d}^{T} \dot{\widehat{\tau}}_{i d}\right) \\
& +\frac{a_{i j}}{2} \sum_{i=1}^{n} \sum_{j=1}^{n}\left(2 \sigma_{i}^{T} d_{j i}^{2} \sigma_{j}\left(t-T_{j i}\right)-\sigma_{i}^{T} d_{j i}^{2} \sigma_{i}\right) \\
& -\frac{a_{i j}}{2} \sum_{i=1}^{n} \sum_{j=1}^{n} d_{j i}^{2} \sigma_{j}^{T}\left(t-T_{j i}\right) \sigma_{j}\left(t-T_{j i}\right) .
\end{aligned}
$$

Choose the adaptive control law of $\widehat{\tau}_{i d}$ is

$$
\dot{\widehat{\tau}}_{i d}=\kappa_{i} M_{i}^{-T} \sigma_{i}
$$

Then, (36) yields

$$
\begin{aligned}
\dot{V}_{3} \leq & -\sum_{i=1}^{n}\left(\tilde{\eta}_{i}^{T} \Lambda \tilde{\eta}_{i}+\sigma_{i}^{T} \operatorname{sign}\left(\sigma_{i}\right)\right) \\
& -\frac{a_{i j}}{2} \sum_{i=1}^{n} \sum_{j=1}^{n} d_{j i}^{2}\left(\sigma_{i}-\sigma_{j}\left(t-T_{j i}\right)\right)^{T}\left(\sigma_{i}-\sigma_{j}\left(t-T_{j i}\right)\right) \\
\leq & -\sum_{i=1}^{n}\left(\tilde{\eta}_{i}^{T} \Lambda \tilde{\eta}_{i}+\left|\sigma_{i}\right|\right) \\
& -\frac{a_{i j}}{2} \sum_{i=1}^{n} \sum_{j=1}^{n} d_{j i}^{2}\left(\sigma_{i}-\sigma_{j}\left(t-T_{j i}\right)\right)^{T}\left(\sigma_{i}-\sigma_{j}\left(t-T_{j i}\right)\right) .
\end{aligned}
$$

Hence, $\widetilde{\eta}_{i}, \sigma_{i}, \sigma_{i}-\sigma_{j}\left(t-T_{j i}\right)$ are bounded, and $\tilde{\eta}_{i} \rightarrow 0$, $\sigma_{i} \rightarrow 0,\left(\sigma_{i}-\sigma_{j}\left(t-T_{j i}\right)\right) \rightarrow 0$, as $t \rightarrow \infty$. The delayed synchronization can be further rewritten as

$$
\begin{aligned}
\sigma_{i}-\sigma_{j}\left(t-T_{j i}\right)= & \dot{\eta}_{i}-\dot{\eta}_{i d}+\Lambda\left(\eta_{i}-\eta_{i d}\right)-\dot{\eta}_{j}\left(t-T_{j i}\right) \\
& +\dot{\eta}_{j d}\left(t-T_{j i}\right) \\
& -\Lambda\left(\eta_{j}\left(t-T_{j i}\right)-\eta_{j d}\left(t-T_{j i}\right)\right) \\
= & \dot{\eta}_{i}-\dot{\eta}_{j}\left(t-T_{j i}\right)+\dot{\eta}_{j d}\left(t-T_{j i}\right)-\dot{\eta}_{i d} \\
& +\Lambda\left(\eta_{i}-\eta_{j}\left(t-T_{j i}\right)+\eta_{j d}\left(t-T_{j i}\right)-\eta_{i d}\right) \\
= & \dot{e}_{i j}+\Lambda e_{i j},
\end{aligned}
$$

where $e_{i j}=\eta_{i}-\eta_{j}\left(t-T_{j i}\right)+\eta_{j d}\left(t-T_{j i}\right)-\eta_{i d}$; similar to the preceding section, we can approve that the trajectory tracking error, velocity tracking error, and synchronization error are asymptotically stable.

Theorem 2. Consider a group of $n$ vessels described by (7) with communication delay in the communication network, using the delayed synchronization control law (34) and disturbances adaptive control law (37); the trajectory tracking error, velocity tracking error, and synchronization error are asymptotically stable, and the multiple vessels can realize synchronization movement. 


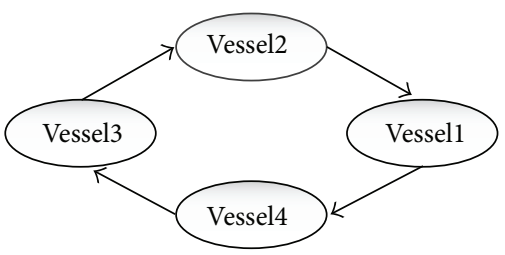

(a)

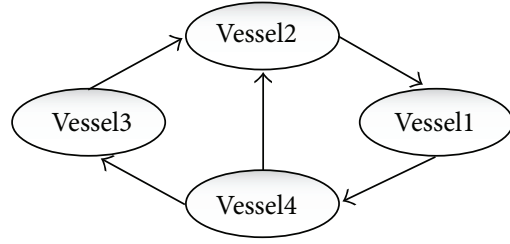

(b)

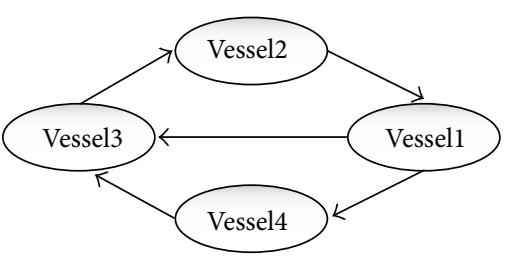

(c)

FIGURE 1: The communication topologies of vessels.

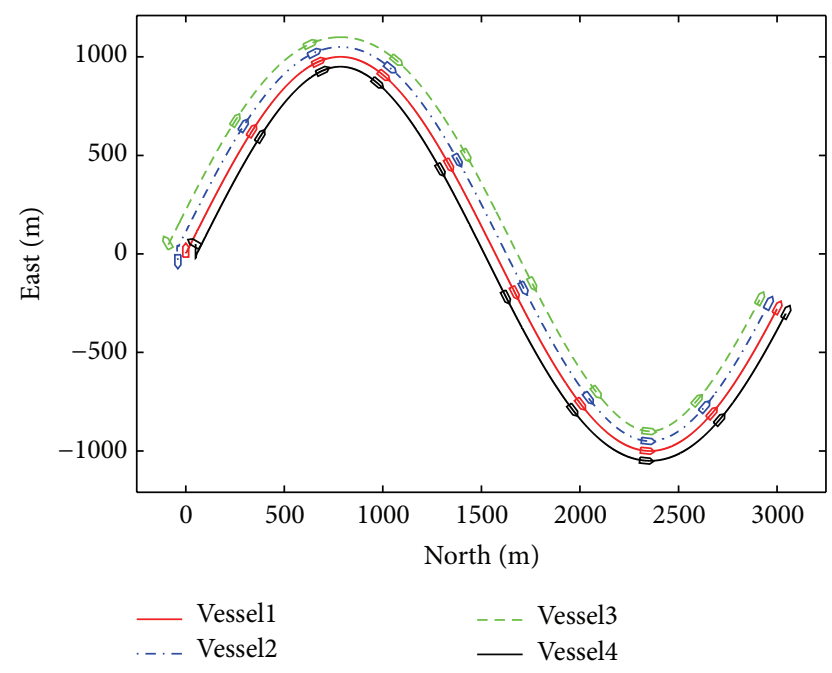

FIGURE 2: Synchronization movement curves of vessels.

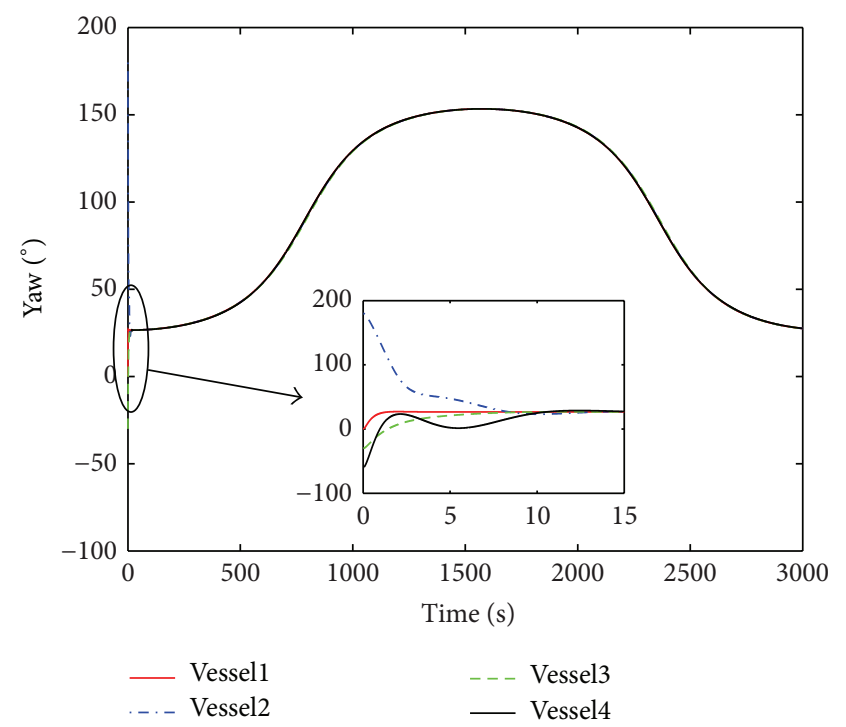

Figure 3: The heading yaw curves of vessels.

\section{Simulation Results}

To verify the effectiveness of the proposed synchronization control algorithm, some simulations are carried out. Assume

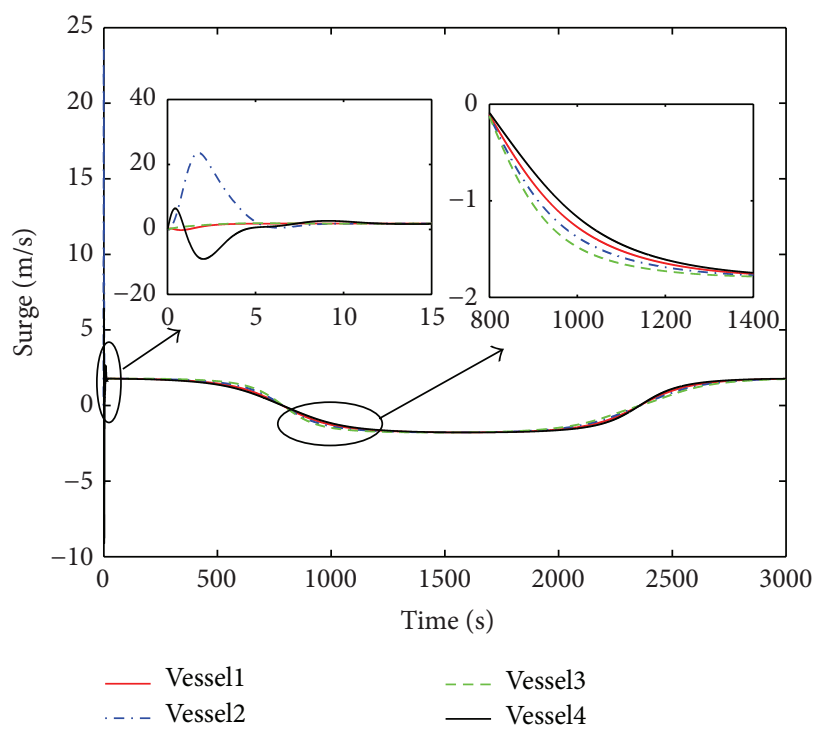

FIGURE 4: The surge velocity curves of vessels.

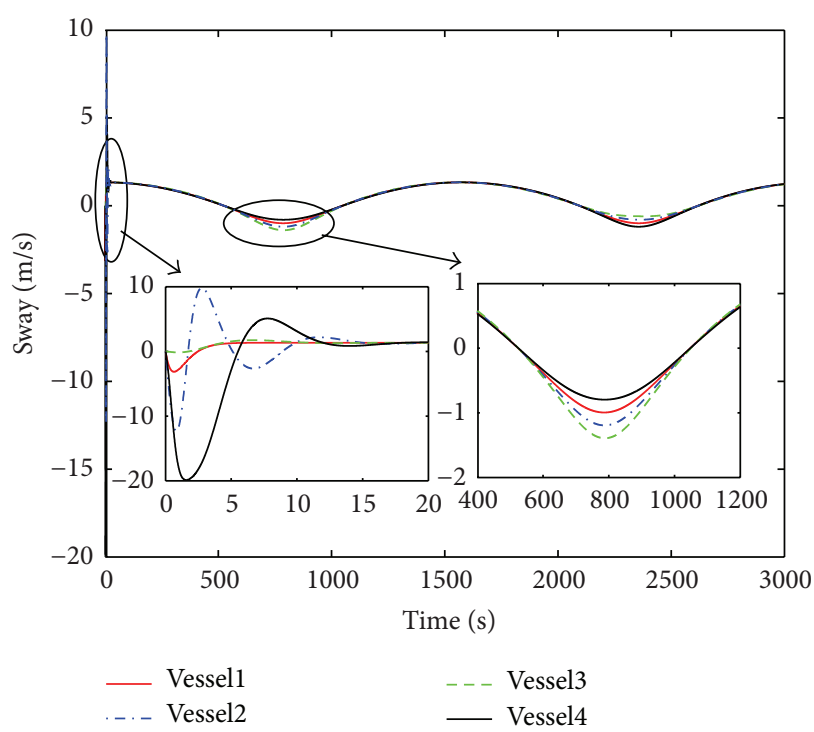

Figure 5: The sway velocity curves of vessels.

there are four vessels; the mode parameters are illustrated in [29]. The switching topologies are illustrated in Figure 1. 


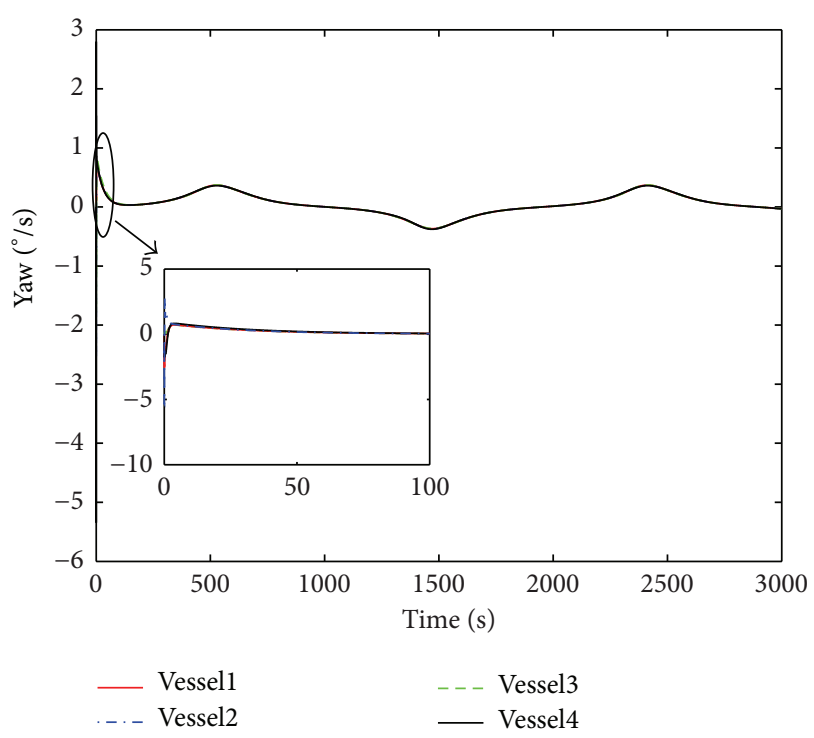

Figure 6: The yaw velocity curves of vessels.

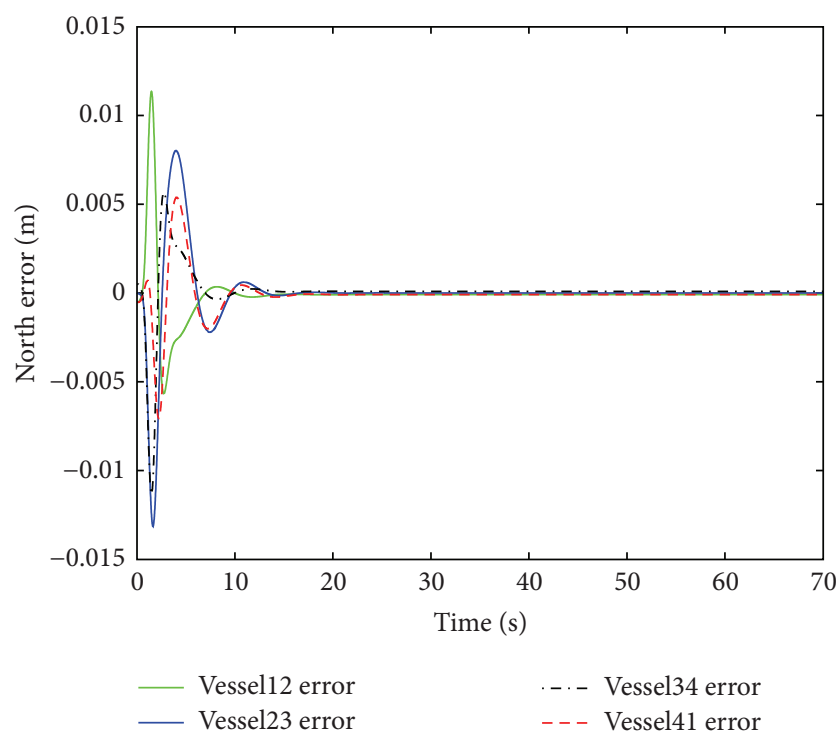

FIGURE 7: Synchronization north position error with time delay.

Therefore, the adjacency matrix and Laplacian matrix of the communication graphs are

$$
\begin{aligned}
& A_{a}=0.5 \times\left[\begin{array}{llll}
0 & 0 & 0 & 1 \\
1 & 0 & 0 & 0 \\
0 & 1 & 0 & 0 \\
0 & 0 & 1 & 0
\end{array}\right], \\
& A_{b}=0.5 \times\left[\begin{array}{llll}
0 & 0 & 0 & 1 \\
1 & 0 & 0 & 0 \\
0 & 1 & 0 & 0 \\
0 & 1 & 1 & 0
\end{array}\right],
\end{aligned}
$$

$$
\begin{gathered}
A_{c}=0.5 \times\left[\begin{array}{llll}
0 & 0 & 1 & 1 \\
1 & 0 & 0 & 0 \\
0 & 1 & 0 & 0 \\
0 & 0 & 1 & 0
\end{array}\right], \\
L_{a}=0.5 \times\left[\begin{array}{cccc}
1 & -1 & 0 & 0 \\
0 & 1 & -1 & 0 \\
0 & 0 & 1 & -1 \\
-1 & 0 & 0 & 1
\end{array}\right], \\
L_{b}=0.5 \times\left[\begin{array}{cccc}
1 & -1 & 0 & 0 \\
0 & 2 & -1 & -1 \\
0 & 0 & 1 & -1 \\
-1 & 0 & 0 & 1
\end{array}\right], \\
L_{c}=0.5 \times\left[\begin{array}{cccc}
1 & -1 & 0 & 0 \\
0 & 1 & -1 & 0 \\
-1 & 0 & 2 & -1 \\
-1 & 0 & 0 & 1
\end{array}\right]
\end{gathered}
$$

Assume the initial position of vessels as follows:

$$
\begin{array}{cc}
\eta_{1}=\left[\begin{array}{lll}
0 & 10 & 0
\end{array}\right]^{T}, \quad \eta_{2}=\left[\begin{array}{lll}
-40 & 30 & \pi
\end{array}\right]^{T}, \\
\eta_{3}=\left[\begin{array}{lll}
-90 & 50 & \frac{\pi}{6}
\end{array}\right]^{T}, & \eta_{4}=\left[\begin{array}{lll}
50 & -10 & \frac{\pi}{3}
\end{array}\right]^{T} .
\end{array}
$$

And the desired trajectory of each vessel is

$$
\begin{gathered}
\eta_{i d}=\left[\begin{array}{lll}
n_{i d} & e_{i d} & \psi_{i d}
\end{array}\right]^{T}, \quad \psi_{i d}=\operatorname{artan} \frac{\dot{n}_{i d}}{\dot{e}_{i d}}, \\
n_{1 d}=t, \quad e_{1 d}=1000 \sin \frac{t}{500}, \quad n_{2 d}=t, \\
e_{2 d}=1000 \sin \frac{t}{500}+R\left(\psi_{1 d}\right) \times\left[\begin{array}{lll}
0 & 50 & 0
\end{array}\right]^{T}, \\
n_{3 d}=t, \\
e_{3 d}=1000 \sin \frac{t}{500}+R\left(\begin{array}{ll}
\psi_{1 d}
\end{array}\right) \times\left[\begin{array}{lll}
0 & 100 & 0
\end{array}\right]^{T}, \\
n_{4 d}=t, \\
e_{4 d}=1000 \sin \frac{t}{500}+R\left(\psi_{1 d}\right) \times\left[\begin{array}{lll}
0 & -50 & 0
\end{array}\right]^{T} .
\end{gathered}
$$

The external disturbances are chosen as

$$
\tau_{i d}=\left[2 \sin \left(\frac{t}{100}\right) \sin \left(\frac{t}{150}\right)-1 \sin \left(\frac{t}{100}\right)+1\right]^{T} .
$$

The parameters of the controller are

$$
\kappa_{i}=3, \quad \Lambda=\operatorname{diag}(10,15,8) .
$$




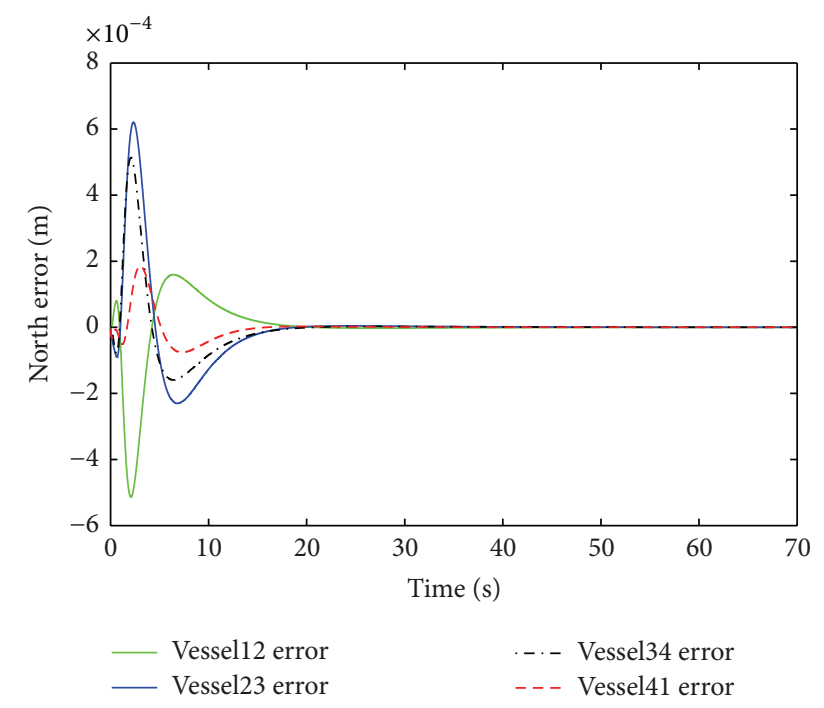

FIGURE 8: Synchronization north position error without time delay.

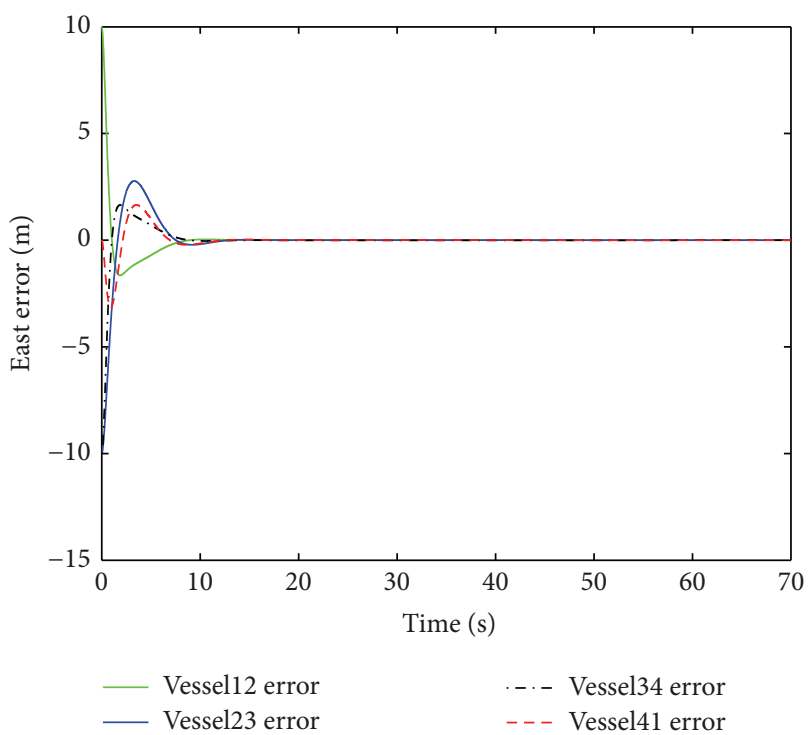

FIGURE 9: Synchronization east position error with time delay.

The simulation results with switching topologies are shown in Figures 2-6. In the first $1000 \mathrm{~s}$, communication topology (a) is used, and from $1000 \mathrm{~s}$ to $2000 \mathrm{~s}$, the communication topology (b) is employed. In the last $1000 \mathrm{~s}$, the communication topology (c) is employed.

The synchronization movement curves of vessels are shown in Figure 2. From the curves, it can be seen that the proposed control law can achieve synchronization movement for multiple vessels in the presence of switching communication topologies. The heading yaw curves of four vessels are shown in Figure 3. And in Figures 4, 5, and 6, the surge velocity, sway velocity, and yaw velocity in the synchronization process are shown.

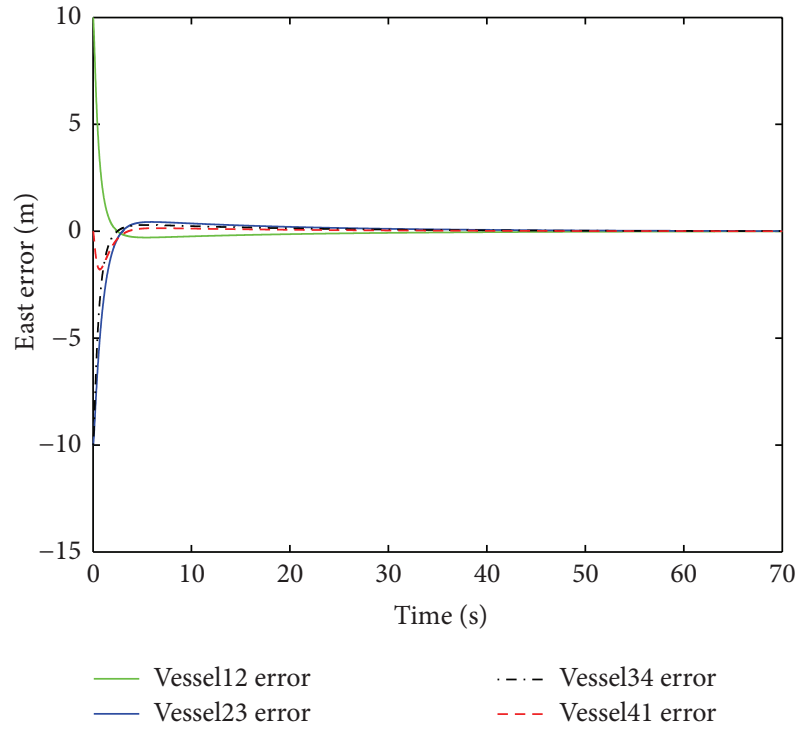

FIGURE 10: Synchronization east position error without time delay.

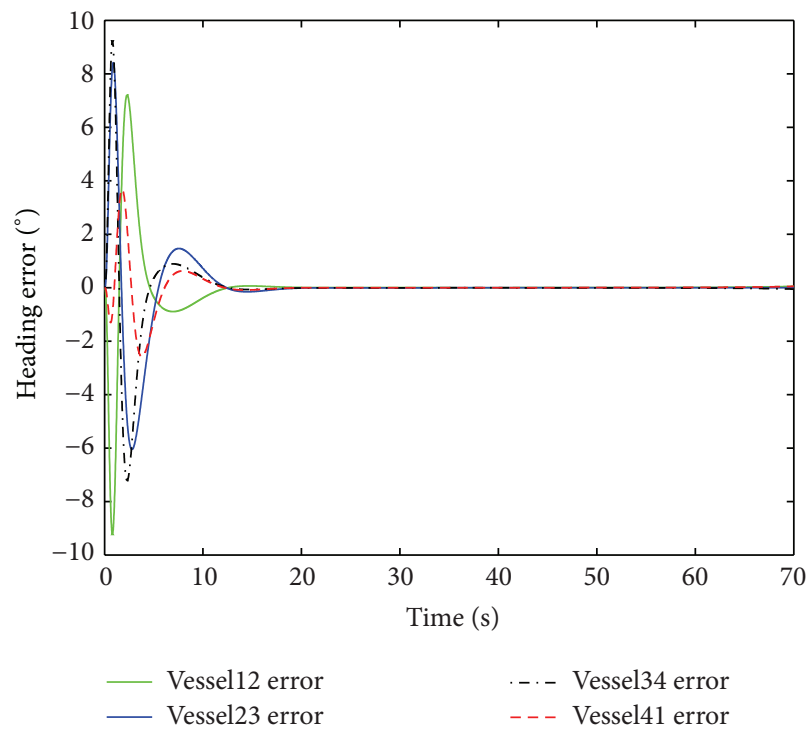

FIGURE 11: Synchronization heading error with time delay.

In the next section simulation, we discuss when there is time-varying time delay in the communication topology (a), and simulations are carried out. Assume the time delay as follows: $T_{14}=T_{21}=0.1+0.3 \sin (t / 10) T_{43}=$ $T_{32}=0.2 \sin (t / 5), d_{j i}$ is assumed to be equal for the sake of simplicity which is selected as $d_{j i}=0.4$. The simulation results are shown in Figures 7, 8, 9, 10, 11, 12, 13, 14, 15, 16, 17, and 18.

From Figures 7-18, it can be seen that comparing to the case without time delay in the communication network, the adjusting time may be longer. But the synchronization 


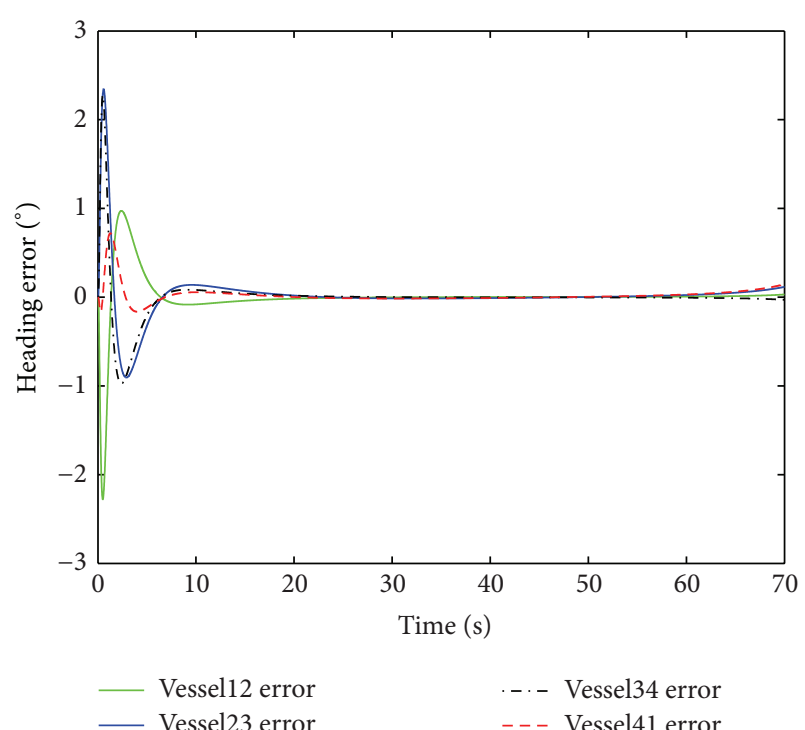

FIGURE 12: Synchronization heading error without time delay.

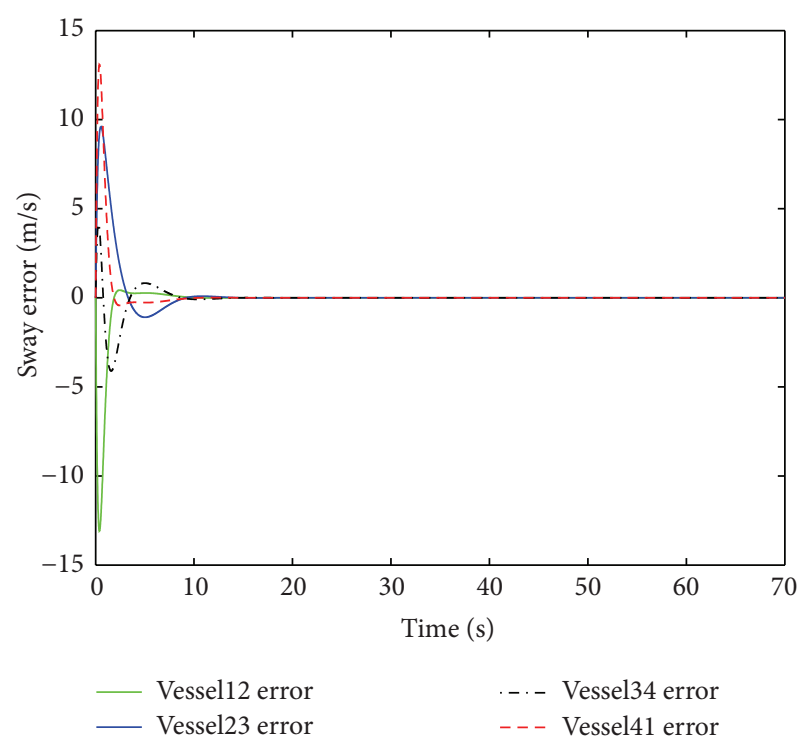

FIGURE 13: Synchronization sway velocity error with time delay.

position error and velocity error among vessels asymptotically converge to zeros in the presence of time delay; that is, the vessels can achieve synchronization movement, and the designed control law is robust to time-varying time delay.

\section{Conclusion}

For the synchronization control of multiple vessels in the presence of switching communication topologies, an adaptive synchronization control algorithm is proposed. With the directed connected graph describing the communication

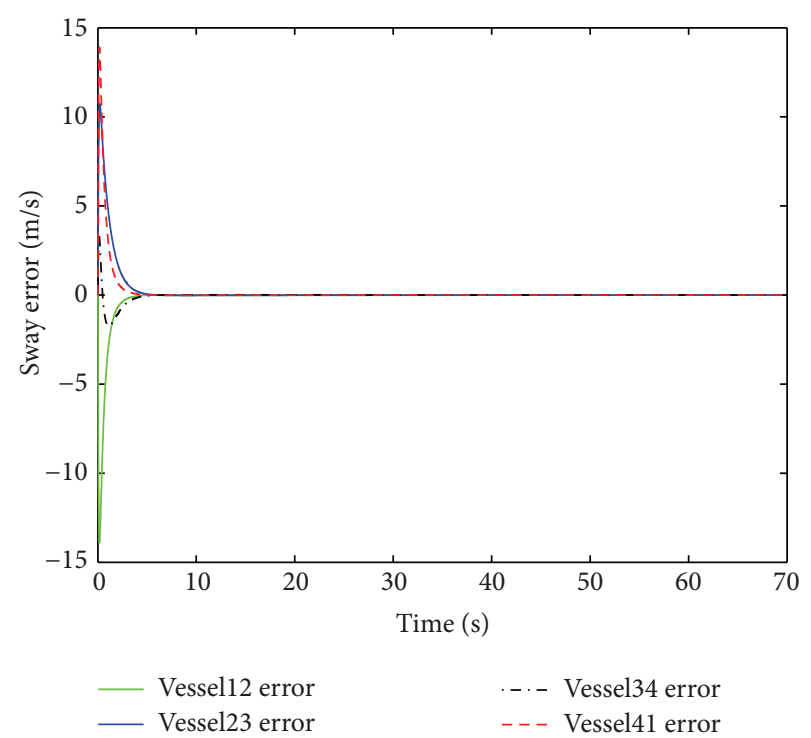

FIGURE 14: Synchronization sway velocity error without time delay.

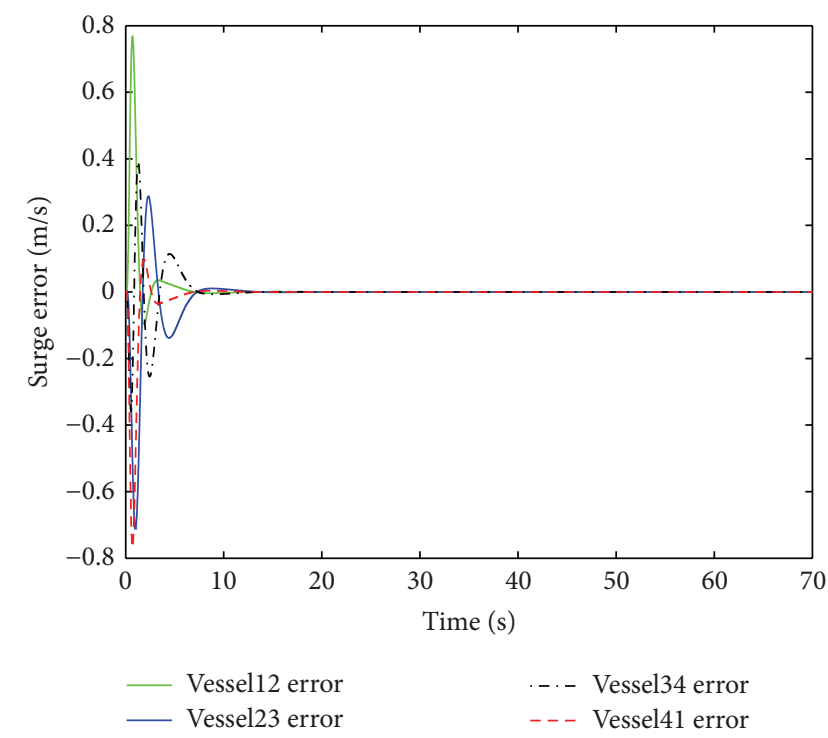

FIGURE 15: Synchronization surge velocity error with time delay.

topology among vessels, a new cross coupling error that includes trajectory tracking error and velocity tracking error is added into the backstepping sliding mode controller, and an adaptive term is introduced to estimate the unknown external disturbances. Furthermore, by introducing a new parameter, the improved synchronization control law is robust to time-varying time delay. Finally, the effectiveness of the synchronization control strategy is supported through several simulations. In the future work, it is desired to consider the mode uncertainty, and it would be useful considering that the velocities of vessels are unknown. 


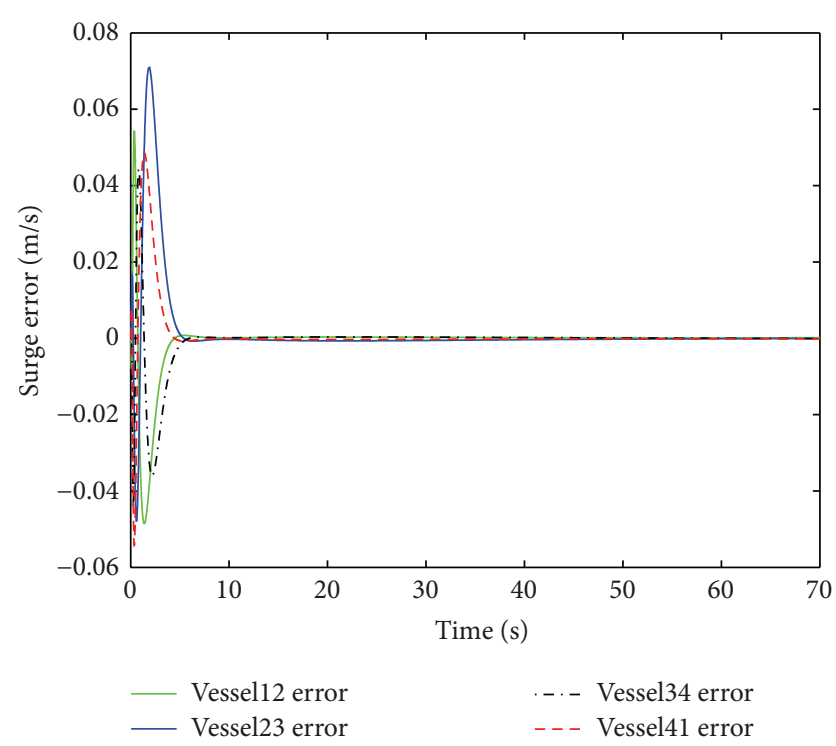

FIGURE 16: Synchronization surge velocity error without time delay.

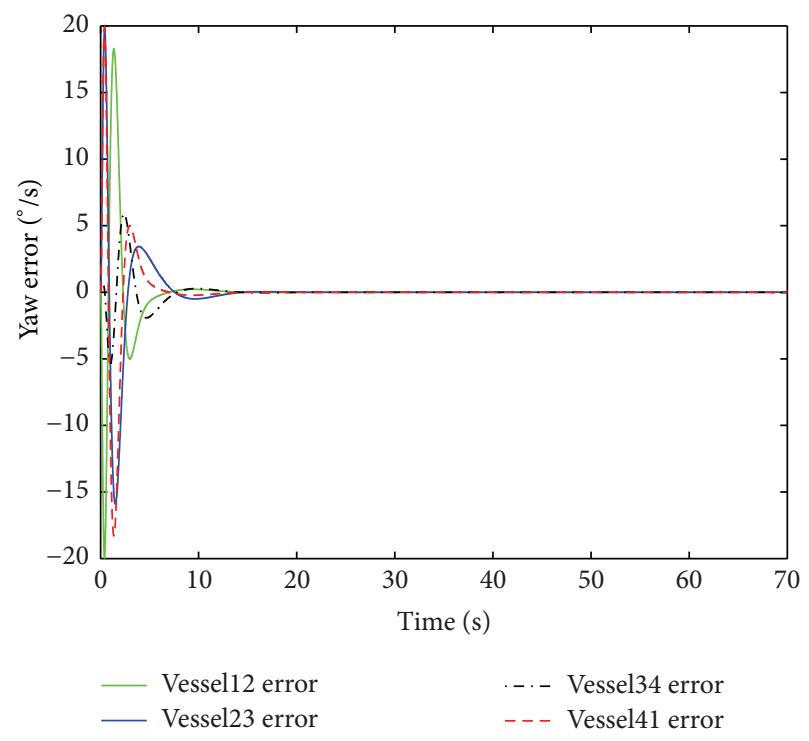

FIGURE 17: Synchronization yaw velocity error with time delay.

\section{Conflict of Interests}

The authors declare that there is no conflict of interests regarding the publication of this paper.

\section{Acknowledgments}

The work is supported by the National Natural Science Foundation of China (no. 51209056) and the Fundamental Research Funds for the Central Universities (no. HEUCF041401).

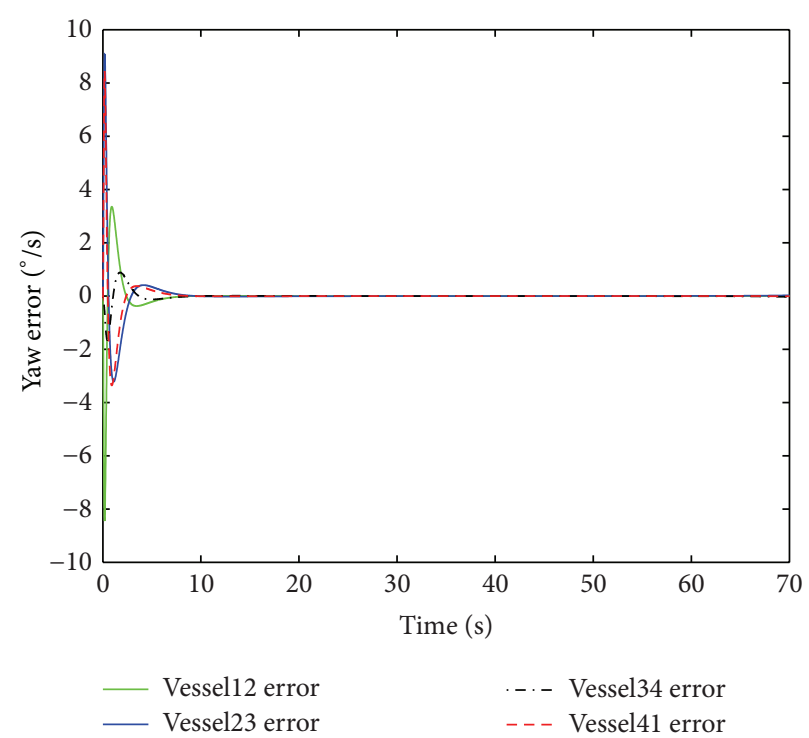

FIGURE 18: Synchronization yaw velocity error without time delay.

\section{References}

[1] S.-J. Chung and J.-J. E. Slotine, "Cooperative robot control and concurrent synchronization of lagrangian systems," IEEE Transactions on Robotics, vol. 25, no. 3, pp. 686-700, 2009.

[2] Y. Bouteraa, J. Ghommam, N. Derbel, and G. Poisson, "Nonlinear control and synchronization with time delays of multiagent robotic systems," Journal of Control Science and Engineering, vol. 2011, Article ID 632374, 10 pages, 2011.

[3] G. A. Leonov, A. I. Bunin, and N. Koksch, "Attraktorlokalisierung des Lorenz-Systems," Zeitschrift für Angewandte Mathematik und Mechanik, vol. 67, no. 12, pp. 649-656, 1987.

[4] T. Zhou, J. Lü, G. Chen, and Y. Tang, "Synchronization stability of three chaotic systems with linear coupling," Physics Letters A, vol. 301, no. 3-4, pp. 231-240, 2002.

[5] A.-M. Zou, K. D. Kumar, and Z.-G. Hou, "Attitude coordination control for a group of spacecraft without velocity measurements," IEEE Transactions on Control Systems Technology, vol. 20, no. 5, pp. 1160-1174, 2012.

[6] R. W. Beard, J. Lawton, and F. Y. Hadaegh, "A coordination architecture for spacecraft formation control," IEEE Transactions on Control Systems Technology, vol. 9, no. 6, pp. 777-790, 2001.

[7] W. Ren, "Distributed cooperative attitude synchronization and tracking for multiple rigid bodies," IEEE Transactions on Control Systems Technology, vol. 18, no. 2, pp. 383-392, 2010.

[8] H. Wang, "Passivity based synchronization for networked robotic systems with uncertain kinematics and dynamics," Automatica, vol. 49, no. 3, pp. 755-761, 2013.

[9] D. Sun, "Position synchronization of multiple motion axes with adaptive coupling control," Automatica, vol. 39, no. 6, pp. 997$1005,2003$.

[10] H. Du and S. Li, "Attitude synchronization control for a group of flexible spacecraft," Automatica, vol. 50, no. 2, pp. 646-651, 2014.

[11] S.-J. Chung and J.-J. E. Slotine, "Cooperative robot control and synchronization of lagrangian systems," in Proceedings of the 
46th IEEE Conference on Decision and Control (CDC '07), pp. 2504-2509, New Orleans, La, USA, December 2007.

[12] Z. Peng, D. Wang, Z. Chen, X. Hu, and W. Lan, "Adaptive dynamic surface control for formations of autonomous surface vehicles with uncertain dynamics," IEEE Transactions on Control Systems Technology, vol. 21, no. 2, pp. 513-520, 2013.

[13] D. Zhao, T. Zou, S. Li, and Q. Zhu, "Adaptive backstepping sliding mode control for leader-follower multi-agent systems," IET Control Theory \& Applications, vol. 6, no. 8, pp. 1109-1117, 2012.

[14] B. A. Idowu, U. E. Vincent, and A. N. Njah, "Generalized adaptive backstepping synchronization for non-identical parametrically excited systems," Nonlinear Analysis. Modelling and Control, vol. 14, no. 2, pp. 165-176, 2009.

[15] J. Ghommam, M. Saad, and F. Mnif, "Robust adaptive formation control of fully actuated marine vessels using local potential functions," in Proceedings of the IEEE International Conference on Robotics and Automation (ICRA '10), pp. 3001-3007, May 2010.

[16] J. Hu and Y. Hong, "Leader-following coordination of multiagent systems with coupling time delays," Physica A: Statistical Mechanics and Its Applications, vol. 374, no. 2, pp. 853-863, 2007.

[17] E. Peymani and T. I. Fossen, "Leader-follower formation of marine craft using constraint forces and lagrange multipliers," in Proceedings of the 51st IEEE Conference on Decision and Control, pp. 2447-2452, December 2012.

[18] A. Jadbabaie, J. Lin, and A. S. Morse, "Coordination of groups of mobile autonomous agents using nearest neighbor rules," IEEE Transactions on Automatic Control, vol. 48, no. 6, pp. 988-1001, 2003.

[19] W. Ren and R. W. Beard, "Consensus seeking in multiagent systems under dynamically changing interaction topologies," IEEE Transactions on Automatic Control, vol. 50, no. 5, pp. 655661, 2005.

[20] Y. Bouteraa, J. Ghommam, N. Derbel, and G. Poisson, "Nonlinear adaptive synchronisation control of multi-agent robotic systems," International Journal of Systems, Control and Communications, vol. 4, no. 1-2, pp. 55-71, 2012.

[21] H. Zhang, K. Pothuvila, Q. Hui, R. Yang, and J. M. Berg, "Control of synchronization for multi-agent systems in acceleration motion with additional analysis of formation control," in Proceedings of the American Control Conference (ACC '11), pp. 509-514, San Francisco, Calif, USA, July 2011.

[22] A. Das and F. L. Lewis, "Cooperative adaptive control for synchronization of second-order systems with unknown nonlinearities," International Journal of Robust and Nonlinear Control, vol. 21, no. 13, pp. 1509-1524, 2011.

[23] A. R. Mehrabian, S. Tafazoli, and K. Khorasani, "Cooperative tracking control of euler-lagrange systems with switching communication network topologies," in Proceedings of the IEEE/ASME International Conference on Advanced Intelligent Mechatronics, pp. 756-762, 2010.

[24] H. Shi, L. Wang, and T. Chu, "Coordinated control of multiple interactive dynamical agents with asymmetric coupling pattern and switching topology," in Proceedings of the IEEE/RSJ International Conference on Intelligent Robots and Systems (IROS '06), pp. 3209-3214, Beijing, China, October 2006.

[25] Y.-C. Liu and N. Chopra, "Robust controlled synchronization of interconnected robotic systems," in Proceedings of the American Control Conference (ACC '10), pp. 1434-1439, Baltimore, Md, USA, July 2010.
[26] S. Yin, H. Luo, and S. X. Ding, "Real-time implementation of fault-tolerant control systems with performance optimization," IEEE Transactions on Industrial Electronics, vol. 61, no. 5, pp. 2402-2411, 2014.

[27] I. A. Gravagne, J. M. Davis, and J. J. Dacunha, "A unified approach to high-gain adaptive controllers," Abstract and Applied Analysis, vol. 2009, Article ID 198353, 8 pages, 2009.

[28] W. Zhang, Z. Wang, and Y. Guo, "Adaptive backstepping-based synchronization of uncertain networked Lagrangian systems," in Proceedings of the American Control Conference (ACC '11), pp. 1057-1062, San Francisco, Calif, USA, July 2011.

[29] E. Kyrkjebø and K. Y. Pettersen, Motion coordination of mechanical systems [Ph.D. thesis], Department of Engineering Cybernetics, Norwegian University of Science and Technology, 2007. 


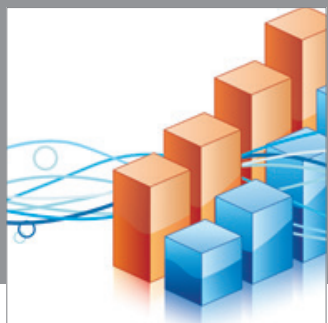

Advances in

Operations Research

mansans

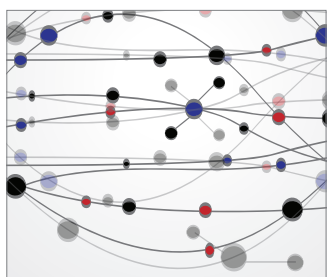

The Scientific World Journal
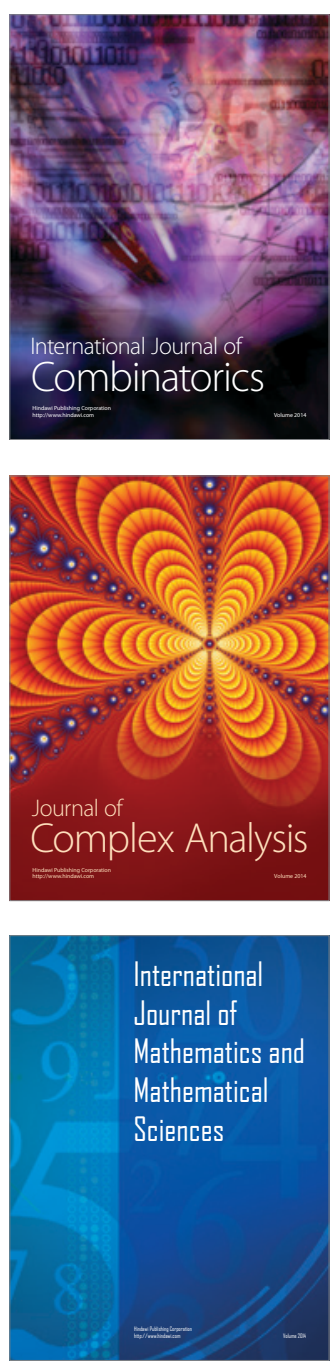
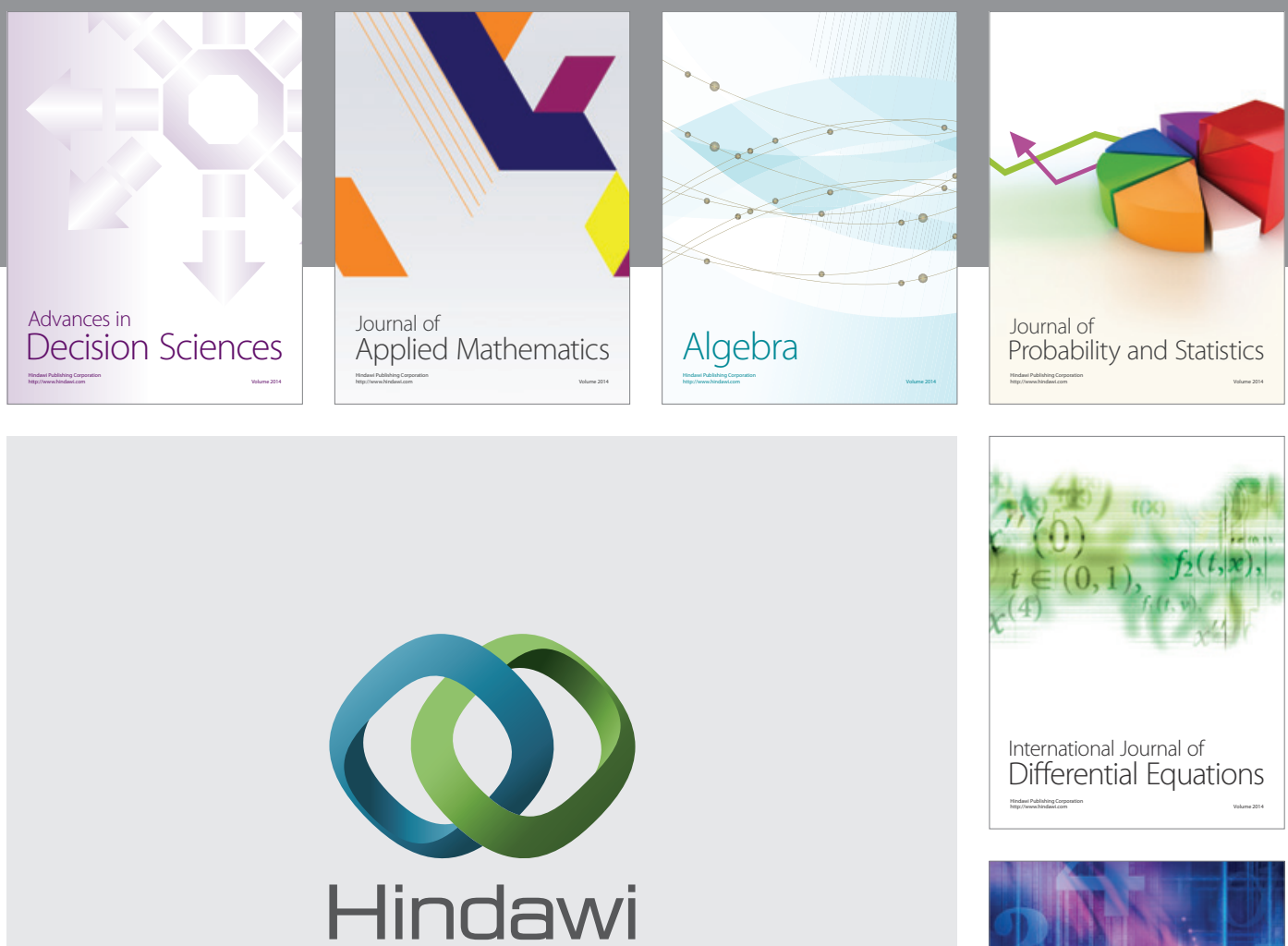

Submit your manuscripts at http://www.hindawi.com
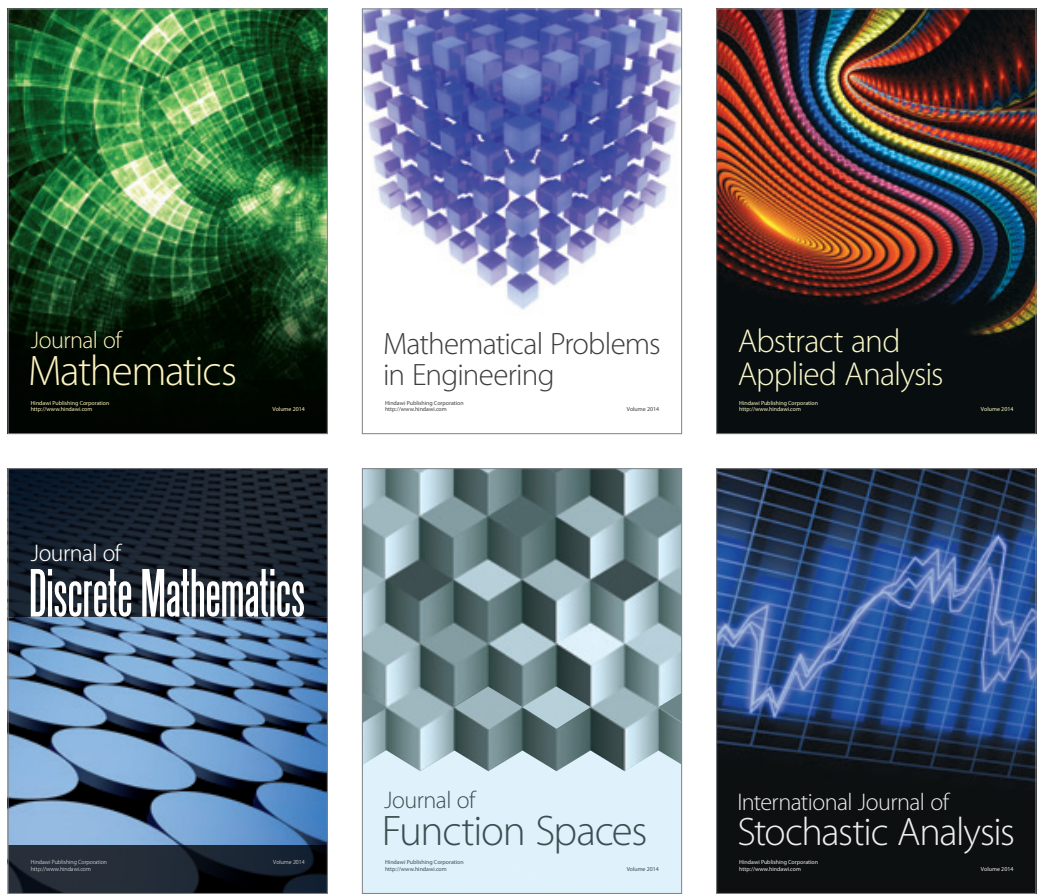

Journal of

Function Spaces

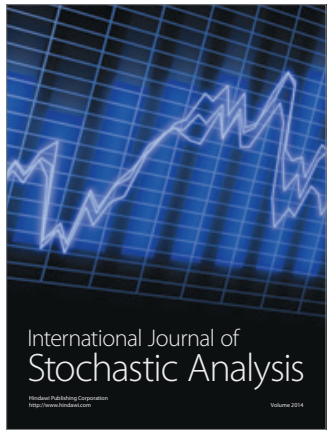

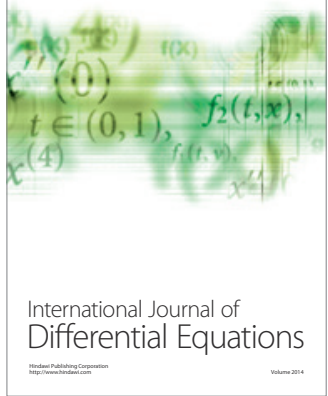
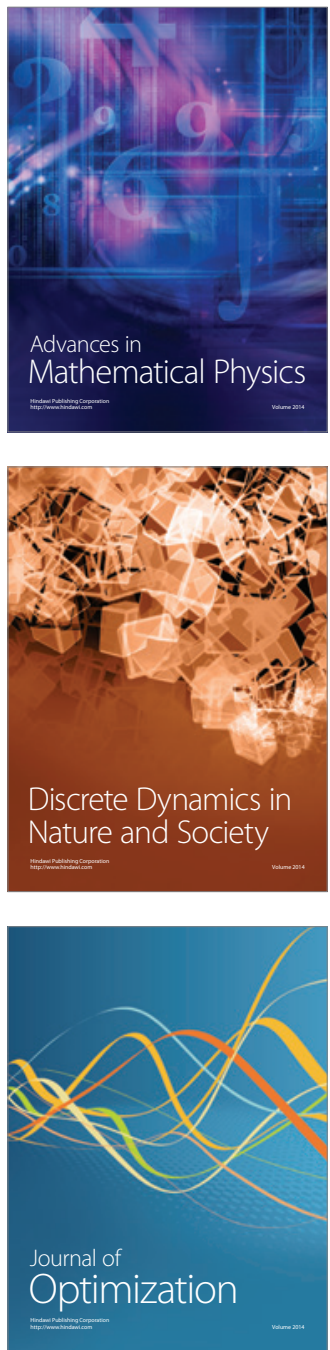